\title{
Censo de servicios de genética médica en Argentina
}

\author{
Rosa Liascovich, ${ }^{1}$ Sandra Rozental, ${ }^{1}$ Pablo Barbero, ${ }^{1}$ Liliana Alba ${ }^{1}$ \\ y Zulma Ortiz ${ }^{2}$
}

Forma de citar Liascovich R, Rozental S, Barbero P, Alba L, Ortiz Z. Censo de servicios de genética médica en Argentina. Rev Panam Salud Publica. 2006;19(2):104-11.

RESUMEN Objetivo. Caracterizar los servicios de genética médica de Argentina, con el propósito de contribuir a la organización de una red que coordine la actividad asistencial, disminuya la inequidad en el acceso y promueva el desarrollo integral de la genética médica en el país.

Métodos. Se realizó un padrón (abril-agosto de 2004) de los servicios de genética médica de Argentina, por medio de una encuesta sobre recursos humanos, procedimientos diagnósticos, utilización de servicios y financiamiento. El criterio de inclusión fue que los servicios realizaran asesoramiento genético o pruebas diagnósticas citogenéticas, de biología molecular o bioquímicas para la detección de defectos congénitos o enfermedades genéticas. No se consideraron los centros dedicados exclusivamente a la investigación.

Resultados. Cumplieron el criterio de inclusión 134 servicios. De ellos, 84 eran servicios privados y los demás 50 eran servicios públicos en universidades y hospitales. Trabajaban en los servicios públicos un total de 67 médicos con formación en genética y 133 profesionales de laboratorio. La mayor parte de los servicios realizaban diagnóstico clínico y citogenético, con un menor desarrollo del diagnóstico molecular, bioquímico y prenatal. La región Centro tuvo más de $70 \%$ de los servicios y de los recursos humanos. En 10 de 23 provincias no se identificaron servicios de gestión pública, los cuales se concentran más bien en las grandes ciudades.

Conclusiones. Los servicios existentes muestran una notable heterogeneidad en su distribución geográfica y amplia variabilidad en cuanto a los procedimientos de diagnóstico ofrecidos. La distribución desigual de los servicios es un problema de organización que requiere la aplicación de políticas que garanticen las prestaciones mínimas para la población de todas las regiones del país, así como el acceso a procedimientos diagnósticos de mayor complejidad.

Palabras clave Genética; instalaciones para atención de salud, recursos humanos y servicios; Argentina.

En los últimos decenios los países desarrollados han mostrado una efi-

1 Centro Nacional de Genética Médica, Administración Nacional de Laboratorios e Institutos de Salud (ANLIS), Ministerio de Salud y Ambiente de la Nación, Buenos Aires, Argentina. Toda correspondencia deberá dirigirse a: Rosa Liascovich, Av. Las Heras 2670, 3er. piso, (1425) Ciudad de Buenos Aires, Argentina. Teléfono: 5411-48090799. Correo electrónico: rosalias@genes.gov.ar

2 Centro de Investigaciones Epidemiológicas, Academia Nacional de Medicina, Buenos Aires, Argentina. ciencia creciente en el control de la desnutrición y las enfermedades infectocontagiosas y, al mismo tiempo, un incremento relativo de las afecciones crónicas y degenerativas, en un proceso que se denomina transición epidemiológica. Aunque el mundo subdesarrollado aún se enfrenta a las llamadas enfermedades de la pobreza, en ciertas naciones las enfermedades crónicas y los trastornos genéticos comienzan a ser un problema de salud pública (1).

Los servicios de genética médica son instituciones de salud dedicadas al diagnóstico, asesoramiento, tratamiento y prevención de los defectos congénitos y de todas aquellas enfermedades en las cuales los factores genéticos tienen una participación causal o predisponente. En los últimos dos 
decenios se ha reflexionado mucho sobre la función y organización de los servicios de genética médica en el mundo (1-9). Por lo general, estos servicios tienen médicos con formación en genética clínica, preparados para brindar asesoramiento genético a los afectados y sus familias, así como otros profesionales de salud que no son médicos (bioquímicos, biólogos, etc.) y se encargan de realizar diferentes pruebas diagnósticas de laboratorio. En los servicios de genética el énfasis recae en los dismorfismos, los trastornos del desarrollo y las fallas reproductivas, que suelen implicar estudios citogenéticos y el diagnóstico prenatal de anomalías cromosómicas. Estos servicios suelen incorporar también laboratorios dedicados al diagnóstico bioquímico de errores congénitos del metabolismo, así como al diagnóstico molecular de enfermedades genéticas mediante el análisis directo del ADN. Sin embargo, en los países en vías de desarrollo estos laboratorios están aún poco extendidos o diagnostican un número limitado de afecciones.

Un componente crucial de los servicios de genética es el asesoramiento genético, un proceso de comunicación con el paciente, su familia o ambos durante el cual se transmiten el diagnóstico, la evolución probable del trastorno, su posible tratamiento y la manera en que los mecanismos hereditarios contribuyen al riesgo de recurrencia en futuros hijos. Asimismo, durante este proceso se plantean las diferentes opciones para hacer frente al riesgo de reaparición, a fin de que la familia pueda adoptar el curso de acción que esté más en armonía con sus metas familiares y con sus normas éticas y religiosas. Al mismo tiempo, los servicios de genética también atienden a las necesidades médicas y psicosociales de los pacientes y sus familias. Por otra parte, entre los objetivos de los servicios de genética destacan el desarrollo de acciones de prevención primaria, secundaria y terciaria en la población de referencia, y las actividades de docencia e investigación.

La variabilidad en las características de los servicios de genética y la falta de mecanismos que garanticen su funcio- namiento coordinado, pueden generar, además de un aumento en las brechas de inequidad en el acceso, una ineficiencia en la utilización de recursos. El presente trabajo se planteó el objetivo de caracterizar los servicios de genética médica de Argentina con la finalidad de contribuir a la organización de una red que coordine la actividad asistencial, disminuya la inequidad en el acceso y promueva el desarrollo integral de la genética médica en el país.

\section{MATERIALES Y MÉTODOS}

Para identificar los servicios de genética de Argentina se hizo una revisión de los directorios de genetistas expertos de diferentes instituciones ${ }^{3} \mathrm{y}$ se consultó a los principales hospitales pediátricos y maternidades del país, así como a las oficinas de epidemiología provinciales. El criterio de inclusión fue que los servicios realizaran asesoramiento genético o pruebas diagnósticas citogenéticas, de biología molecular o bioquímicas, para la detección de defectos congénitos o enfermedades genéticas. No se incluyeron los centros dedicados exclusivamente a la investigación que no atendían pacientes.

El registro se realizó mediante una encuesta de tres partes. La primera, un cuestionario enviado por correo electrónico, contenía preguntas para la identificación de los servicios. La segunda parte, una encuesta telefónica, indagó acerca de los procedimientos diagnósticos; la procedencia geográfica de los pacientes; el número de personas atendidas cada mes y las fuentes de financiamiento del servicio. Todas las preguntas, de respuesta cerrada,

\footnotetext{
Sección de Genética Humana de la Sociedad Argentina de Genética; Hospital de Pediatría "Profesor Dr. Juan P. Garrahan"; Hospital de Clínicas "José de San Martín"; Estudio Colaborativo Latinoamericano de Malformaciones Congénitas (ECLAMC); Hospital de Niños de la Santísima Trinidad; Centro de Educación Médica e Investigaciones Clínicas (CEMIC); Clínica FLENI; Academia Nacional de Medicina; Fundación Genos; Laboratorio y Fundación de Enfermedades Neurometabólicas.
}

fueron realizadas por cuatro entrevistadores capacitados para tal fin. La tercera parte, otro cuestionario enviado por correo electrónico, registró los recursos humanos y las actividades de docencia e investigación realizadas en los últimos cinco años en cada servicio. Las preguntas fueron de tipo abierto, con opción a sugerencias y comentarios.

La encuesta se realizó entre el 1 de abril y el 31 de agosto de 2004. Todas las preguntas se tabularon en una base de datos (con el programa EPI-Info, versión 6.4) y las respuestas inespecíficas se excluyeron del análisis. Se utilizaron instrumentos de estadística descriptiva para organizar y presentar los datos. El análisis de la disponibilidad de recursos abarcó las cinco regiones del país: Centro; Cuyo; Patagónica; Noroeste y Noreste (10); dentro de la región Centro se discriminaron tres unidades: la Ciudad Autónoma de Buenos Aires, la provincia de Buenos Aires y el resto de las provincias (véanse detalles de las regiones, provincias y distritos autónomos, en el cuadro 1 y la figura 1). Para las diferentes regiones se establecieron tres indicadores: 1) el número de servicios de genética, 2) el número de médicos con formación en genética clínica y 3) el número de profesionales de laboratorio. Para cada región se calculó la disponibilidad relativa de cada uno de los recursos, como la razón entre cada indicador y la población total.

Los procedimientos diagnósticos censados fueron: genética clínica; diagnóstico citogenético (detección de anomalías cromosómicas constitutivas y trastornos hematológicos); diagnóstico prenatal (incluidos el asesoramiento genético, la toma de muestras de tejido placentario o líquido amniótico y los análisis de laboratorio; se excluyó el diagnóstico prenatal exclusivamente ecográfico); diagnóstico molecular de enfermedades genéticas mediante análisis directo del ADN y diagnóstico bioquímico de errores congénitos del metabolismo. Se consideró respuesta positiva la realización del procedimiento diagnóstico por el propio servicio y respuesta negativa la derivación del caso a otra institución. 
CUADRO 1. Servicios de genética de gestión pública. Argentina, 2004

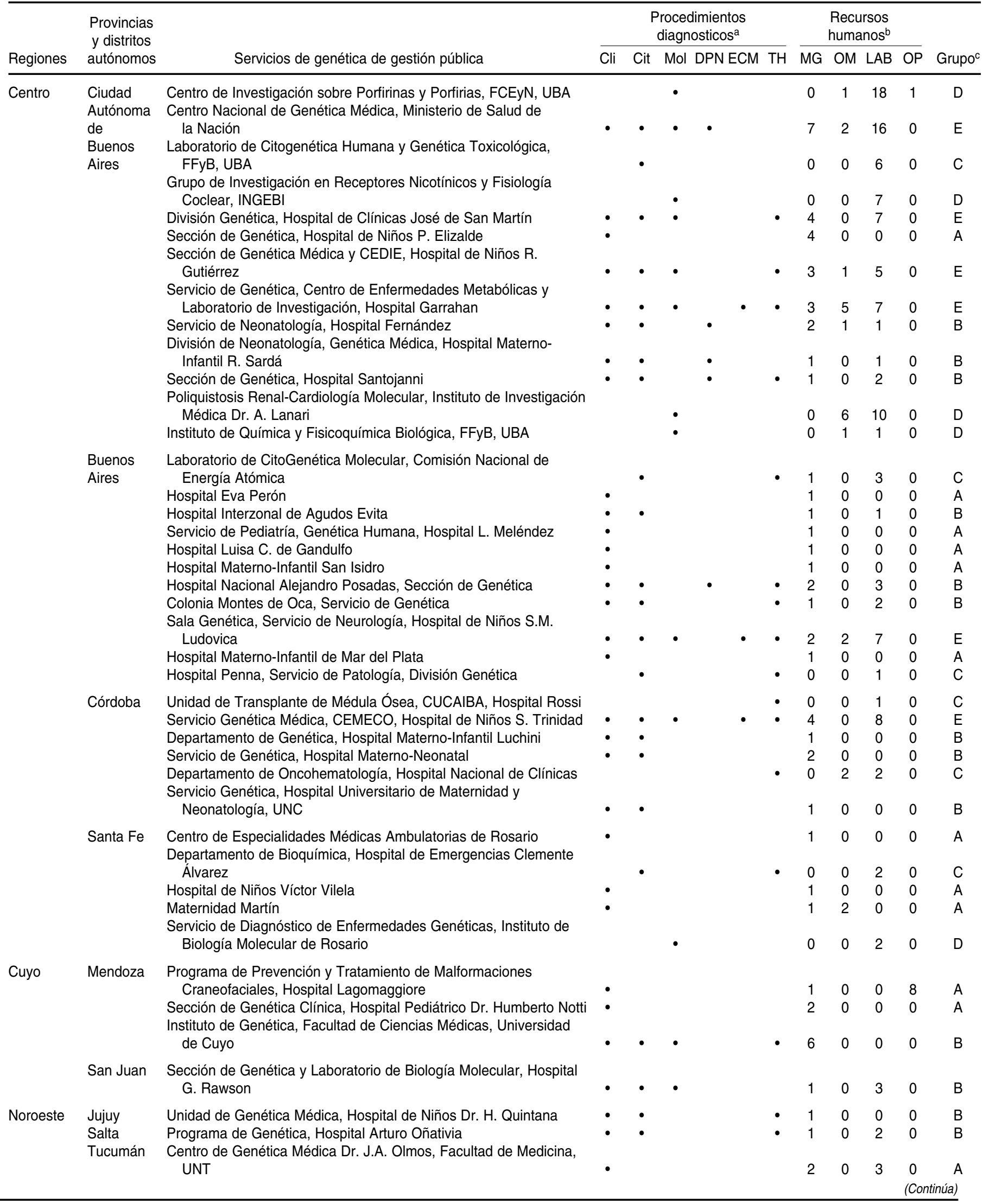


CUADRO 1. Servicios de genética de gestión pública. Argentina, 2004

\begin{tabular}{|c|c|c|c|c|c|c|c|c|c|c|c|c|}
\hline \multirow[b]{2}{*}{ Regiones } & \multirow{2}{*}{$\begin{array}{l}\text { Provincias } \\
\text { y distritos } \\
\text { autónomos }\end{array}$} & \multirow[b]{2}{*}{ Servicios de genética de gestión pública } & \multicolumn{5}{|c|}{$\begin{array}{l}\text { Procedimientos } \\
\text { diagnosticos }^{\mathrm{a}}\end{array}$} & \multicolumn{4}{|c|}{$\begin{array}{l}\text { Recursos } \\
\text { humanos }^{b}\end{array}$} & \multirow[b]{2}{*}{ Grupo $^{\circ}$} \\
\hline & & & Cli & Cit & Mol & DPN ECM & $\mathrm{TH}$ & MG & $\mathrm{OM}$ & LAB & $\mathrm{OP}$ & \\
\hline Noreste & $\begin{array}{l}\text { Corrientes } \\
\text { Misiones }\end{array}$ & $\begin{array}{l}\text { Servicio de Genética, Hospital Pediátrico Juan Pablo II } \\
\text { Hospital Dr. Ramón Madariaga } \\
\text { Laboratorio de Citogenética Humana, Universidad Nacional } \\
\text { de Misiones }\end{array}$ & • & • & $\bullet$ & - & $\bullet$ & $\begin{array}{l}1 \\
0 \\
0\end{array}$ & $\begin{array}{l}0 \\
2 \\
0\end{array}$ & $\begin{array}{l}2 \\
3 \\
5\end{array}$ & $\begin{array}{l}0 \\
1 \\
0\end{array}$ & $\begin{array}{l}\mathrm{B} \\
\mathrm{C} \\
\mathrm{C}\end{array}$ \\
\hline Patagónica & $\begin{array}{l}\text { Neuquén } \\
\text { Río Negro } \\
\text { Santa Cruz }\end{array}$ & $\begin{array}{l}\text { Servicio de Genética, Hospital Castro Rendón } \\
\text { Departamento de Biología, Universidad del Comahue } \\
\text { Hospital Regional de Río Gallegos }\end{array}$ & • & • & & & • & $\begin{array}{l}1 \\
0 \\
1\end{array}$ & $\begin{array}{l}0 \\
0 \\
1\end{array}$ & $\begin{array}{l}0 \\
2 \\
0\end{array}$ & $\begin{array}{l}0 \\
0 \\
0\end{array}$ & $\begin{array}{l}B \\
C \\
A\end{array}$ \\
\hline Total del pa & & & & & & & & 67 & 28 & 133 & 10 & \\
\hline
\end{tabular}

a Cli: genética clínica; Cit: citogenética; Mol: diagnóstico molecular; DPN: diagnóstico prenatal; ECM: errores congénitos metabolismo; TH: trastornos hematológicos.

${ }^{b}$ MG: médicos con formación en genética clínica; OM: otros médicos; LAB: profesionales de laboratorio; OP: otros profesionales.

c Grupo A: consultorios de genética clínica; grupo B: centros de genética clínica y diagnóstico citogenético que en algunos casos también realizan diagnóstico molecular y diagnóstico prenatal; grupo C: laboratorios de citogenética; grupo D: laboratorios de biología molecular; grupo E: centros de mayor complejidad que incluyen genética clínica, citogenética y diagnóstico molecular y que en algunos casos también realizan diagnóstico prenatal y diagnóstico bioquímico de errores congénitos del metabolismo.

Otras abreviaturas: CEDIE, Centro de Investigaciones Endocrinológicas; CEMECO, Centro de Estudios de las Metabolopatías Congénitas; CUCAIBA, Centro Único Coordinador de Ablación e Implantes de Buenos Aires; FCEyN, Facultad de Ciencias Exactas y Naturales; FFyB, Facultad de Farmacia y Bioquímica; INGEBI, Instituto de Investigación en Ingeniería Genética y Biología Molecular; UBA, Universidad de Buenos Aires; UNT, Universidad Nacional de Tucumán.

\section{RESULTADOS}

Se identificaron 165 servicios de genética, de los cuales 134 cumplían con el criterio de inclusión. De los 134 servicios registrados, $84(63 \%)$ fueron de gestión privada y $50(37 \%)$ de gestión pública, dependientes de universidades $\mathrm{u}$ hospitales nacionales, provinciales o municipales. La tasa de respuesta a todo el cuestionario fue de $98 \%$, excepto en lo referente a las preguntas sobre las actividades de docencia e investigación que se desarrollaron en los últimos 5 años, en cuyo caso la cifra fue inferior a $50 \%$, tanto en los servicios de gestión pública como en los de gestión privada. Con respecto a la pregunta abierta sobre las actividades de investigación, los integrantes de los servicios refirieron dificultades para reunir sus antecedentes $\mathrm{y}$, entre los que respondieron, la interpretación de la pregunta fue sumamente heterogénea, con una variedad de respuestas que incluyó desde publicaciones en revistas con diferente impacto, hasta líneas de trabajo sumamente inespecíficas, como "investigación en malformaciones congénitas" o "investigación en retraso mental". Una situación si- milar se observó en la pregunta abierta sobre actividades de docencia, cuyas respuestas incluyeron tanto cursos organizados por los servicios, como clases individuales que algún integrante de estos había impartido. Tales motivos impidieron la evaluación de esas dos preguntas.

Del total de 84 servicios de gestión privada, 57 (68\%) fueron consultorios a cargo de un único médico genetista, o bien pequeños centros, integrados por un médico genetista y un profesional de laboratorio, que atendían hasta 50 pacientes por mes y que estaban dedicados básicamente al diagnóstico clínico y citogenético. Los servicios restantes fueron 21 (25\%) laboratorios (en su mayoría de citogenética y unos cuantos dedicados al diagnóstico molecular), cinco (6\%) centros de mayor envergadura que ofrecían prácticamente todos los procedimientos diagnósticos y un solo centro (1\%) especializado en errores congénitos del metabolismo. Del total de servicios de gestión privada, 73 (87\%) se localizaban en la región Centro y 36 (43\%) realizaban diagnóstico prenatal.

Los resultados que se presentan a continuación se refieren exclusiva- mente a los servicios de gestión pública. Los 50 servicios fueron heterogéneos en cuanto a los procedimientos diagnósticos que realizaban y se clasificaron en cinco grupos según su nivel de complejidad: grupo A, consultorios de genética clínica; grupo $B$, centros de genética clínica y diagnóstico citogenético que en algunos casos también realizan diagnóstico molecular y diagnóstico prenatal; grupo $\mathrm{C}$, laboratorios de citogenética; grupo $\mathrm{D}$, laboratorios de biología molecular, y grupo $\mathrm{E}$, centros de mayor complejidad que abarcan servicios de genética clínica, citogenética y diagnóstico molecular y que en ciertos casos realizan también diagnóstico prenatal y diagnóstico bioquímico de errores congénitos del metabolismo (cuadro 1). La encuesta permitió identificar a un total de 67 médicos con formación en genética clínica; 133 profesionales no médicos capacitados para realizar diferentes pruebas diagnósticas de laboratorio (biólogos, bioquímicos, licenciados en genética, etc.); 28 médicos de otras especialidades (pediatras, tocoginecólogos, neurólogos, etc.) y 10 profesionales de otras áreas de la salud (psicólogos, fonoaudiólogos, nutricionistas, etc.). 
FIGURA 1. Número de servicios de genética de gestión pública en las provincias y distritos autónomos de Argentina



Tres $(6 \%)$ de los servicios funcionaban exclusivamente con personal voluntario (sin remuneración), mientras que los 47 restantes (94\%) estaban financiados por el presupuesto oficial forma privada. Además, 4 servicios (8\%) recibían donaciones de pacientes o asociaciones de padres como fuente habitual de recursos y 11 (22\%) contaban con subsidios internacionales o nacionales para solventar actividades de investigación, que en muchos casos contribuían también a financiar la labor asistencial. En cuanto a la procedencia geográfica de los pacientes, $13(27 \%)$ servicios atendían solo a personas provenientes de la provincia en que se localiza el servicio, mientras que $37(73 \%)$ atendían a un número variable de pacientes procedentes de otras provincias. En cuanto al número de pacientes atendidos, 8 servicios $(16 \%)$ asistían a más de 150 cada mes, $13(26 \%)$ entre 51 y 150, y 29 (58\%) atendían hasta 50 pacientes por mes.

La distribución geográfica de los recursos fue heterogénea: en la región Centro se identificaron $70 \%$ de los servicios, $72 \%$ de los médicos con formación en genética clínica y 85\% de los profesionales de laboratorio. En esta región, la Ciudad Autónoma de Buenos Aires concentró la mayor parte de los recursos, a diferencia de la provincia de Buenos Aires, que mostró una escasez relativa de ellos. El análisis de la distribución de recursos en relación con la población total de cada una de las regiones (cuadro 2) mostró que las diferencias entre estas se atenuaron. El Noreste, el Noroeste y la Patagonia son las regiones que presentaron menor disponibilidad y en esta última, además, los recursos cubren una amplia extensión geográfica. Por otra parte, en 10 provincias no se identificaron servicios de genética de gestión pública y en las 13 provincias restantes la distribución se dio en mayor proporción en las grandes ciudades (figura 1; cuadro 1).

\section{DISCUSIÓN}

En la mayoría de los países de América Latina los servicios de genética muestran escaso desarrollo y, salvo contadas excepciones, no existen programas de base poblacional ni políticas explícitas para la prevención y asistencia de los defectos congénitos (9). Se ha señalado $(1,8)$ que "las deficiencias 


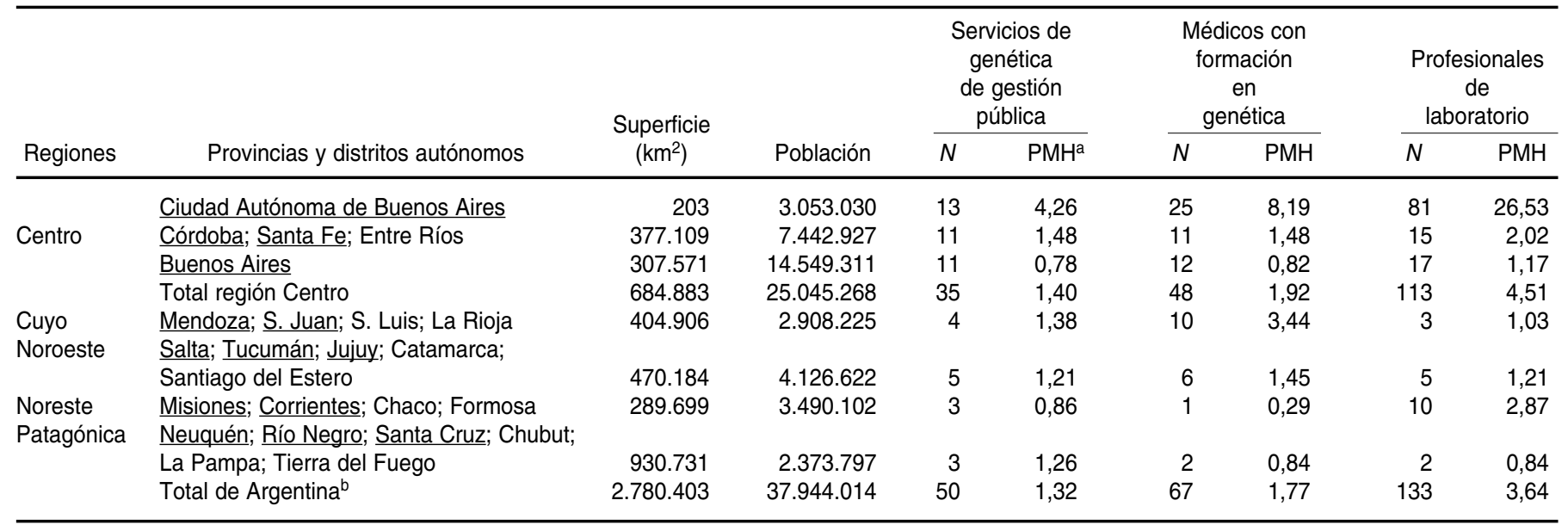

Nota: El subrayado denota las provincias y distritos que cuentan con servicios de genética de gestión pública. No hay estos servicios en las demás entidades.

a PMH, razón por millón de habitantes.

b No incluye el sector Antártico ni las Islas del Atlántico Sur.

que caracterizan a los servicios de genética en los países en desarrollo se explican en parte por: a) la carga de las necesidades no atendidas en otros aspectos de la atención de salud; b) la falta de reconocimiento del carácter prioritario de las afecciones genéticas por parte de los funcionarios de salud y la profesión médica; c) el concepto erróneo de que los servicios de genética son costosos y dedican todo su interés a enfermedades muy poco frecuentes; d) los servicios de genética son de carácter diagnóstico más que terapéutico y en general la prevención se interpreta como la interrupción de los embarazos afectados, y e) en buena medida, el público carece de conocimientos acerca de los riesgos genéticos y las posibilidades de prevención".

Los primeros servicios de genética se establecieron en Argentina a fines del decenio de 1960 y en el de 1970 ya realizaban estudios citogenéticos, con énfasis en el diagnóstico de defectos congénitos y trastornos hematológicos. En el decenio de 1980 algunos servicios comenzaron a ofrecer diagnóstico prenatal de anomalías cromosómicas y en el de 1990 varios centros empezaron a realizar tamizaje prenatal de anomalías fetales utilizando marcadores bioquímicos en el suero materno, y se inició el diagnóstico molecular de enfermedades genéticas mediante el análisis directo del ADN (11). Sin embargo, aunque el desarrollo de la genética médica en el país fue un proceso creciente y continuo, al mismo tiempo se caracterizó por la ausencia de una estrategia planificada. En la actualidad es virtualmente imposible determinar el alcance de la asistencia para defectos congénitos y enfermedades genéticas en Argentina, ya que, por ejemplo, el control prenatal ultrasonográfico, el tamizaje bioquímico prenatal y el diagnóstico de recién nacidos con defectos congénitos, así como la atención de pacientes adultos con enfermedades de causa total o parcialmente genética, se realizan en centros de salud diversos, no siempre articulados con los servicios de genética.

¿Cómo establecer si son adecuados los recursos de genética médica disponibles en el sector público? La prevalencia de defectos congénitos es de aproximadamente $3 \%$ en los recién nacidos (12), aunque se ha señalado que podría alcanzar $8 \%$ a los 5 años de edad (13). En América del Sur, la única fuente de información sobre la frecuencia de malformaciones congénitas en el neonato es el Estudio Colaborativo Latinoamericano de Malformaciones Congénitas (ECLAMC) $(14,15)$, que publicó cifras similares. Sin embargo, en nuestro país no se han creado programas específicos para la detección y registro de los defectos congénitos, y los únicos datos oficiales indican que constituyen la segunda causa de muerte infantil (16). Entre las recomendaciones generales propuestas para los países en desarrollo se ha sugerido contar con un centro de genética de nivel terciario por cada 5 a 10 millones de personas, entendiendo como tal el que brinda una amplia cobertura para el diagnóstico, asesoramiento y atención de los pacientes con trastornos genéticos graves, y lleva a cabo programas de investigación y formación profesional (1). En este trabajo se identificaron 50 servicios de genética de gestión pública, seis de los cuales podrían considerarse de tercer nivel (grupo E). De este modo, dentro de una población total de aproximadamente 38 millones de personas se registró un centro de tercer nivel por cada 6 millones de habitantes, valor similar al sugerido para los países en desarrollo. Al mismo tiempo, la razón hallada de 1,77 médicos con formación en genética clínica por millón de habitantes se aproxima a estimaciones realizadas para la región, ya que se ha señalado que América Latina tiene una distribución cercana a un médico con formación en genética clínica por millón de habitantes (1), en comparación con 2,7 por millón en Europa (6).

Si bien no se descarta la posibilidad de un subregistro de los servicios de 
genética identificados por el presente estudio, tal hecho parece improbable ante la multiplicidad de fuentes utilizadas. Nuestros resultados parecen indicar que en Argentina los recursos no serían insuficientes en términos absolutos, sino que presentarían una amplia variabilidad en los procedimientos de diagnóstico ofrecidos y una notable heterogeneidad geográfica, con especial concentración en la región Centro del país. En lo que atañe a esta región, destaca sobre todo la gran oferta de servicios y de recursos humanos de la Ciudad Autónoma de Buenos Aires. Sin embargo, es preciso aclarar que gran parte de su demanda proviene de pacientes que residen en la provincia de Buenos Aires, de tal manera que, en la práctica, la alta disponibilidad de recursos de la ciudad capital compensa su escasez en la provincia de Buenos Aires, la más populosa del país.

La inequidad en el acceso al diagnóstico y asesoramiento genético se manifiesta en la falta de cobertura para los habitantes de algunas regiones del país, según criterios relativos tanto al tamaño de la población como a las características geográficas (extensión, accesibilidad). En la región Patagónica, por ejemplo, se registraron dos médicos genetistas y dos profesionales de laboratorio para una extensión de casi 1 millón de kilómetros cuadrados y en la región Noreste, un solo médico genetista para una población de casi 3,5 millones. En especial, este estudio mostró un escaso desarrollo del diagnóstico prenatal en el ámbito público, disponible en 36 (43\%) de las 84 instituciones de gestión privada, pero solo en $6(12 \%)$ de los 50 servicios de gestión pública. De este modo, parecería existir una situación sumamente desigual en el acceso a los procedimientos diagnósticos requeridos para las parejas en riesgo, ya que aquellas asistidas en los servicios de gestión privada tendrían mayor probabilidad de tener acceso al diagnóstico prenatal y, en consecuencia, de obtener la información necesaria para decidir la interrupción o no de los embarazos afectados (si bien en Argentina el aborto no es legal y las mujeres recurren a él a su propio riesgo).

Para optimizar los recursos existentes en el país sería apropiado organizar una estructura de red que coordine la derivación de pacientes o las muestras desde los servicios regionales - que deberían garantizar las prestaciones mínimas - hacia los nodos centrales -que deberían contar con laboratorios capaces de ofrecer los procedimientos diagnósticos de mayor complejidad. Esta red de servicios de genética no solamente coordinaría la actividad asistencial, sino que también podría favorecer al desarrollo integral de la genética médica en el país mediante diferentes acciones: el establecimiento de un sistema de control de calidad del servicio, la coordinación de programas de formación de recursos humanos, la realización de proyectos de investigación colaborativos, la garantía de los resguardos bioéticos en la realización de actividades asistenciales y de investigación, la organización de un sistema de registro y vigilancia epidemiológica de defectos congénitos y la coordinación de acciones de prevención de defectos congénitos en la comunidad, entre otras.

Este estudio constituye una primera aproximación hacia el objetivo de sistematizar la información existente. Futuras investigaciones permitirán profundizar in situ en las cualidades específicas de los recursos disponibles, las cuales no se analizaron con la metodología empleada en el presente trabajo.

Agradecimientos. El presente trabajo fue financiado por el Programa Nacional de Vigilancia de la Salud y Control de Enfermedades (VIGIA) del Ministerio de Salud y Ambiente de la Nación. Agradecemos la participación de los servicios de genética censados; a Enrique C. Gadow su colaboración, y a Víctor Penchaszadeh la lectura crítica del original.

\section{REFERENCIAS}

1. Organización Mundial de la Salud y Alianza Mundial de Organizaciones para la Prevención de los Defectos Congénitos (WAOPBD). Reunión Conjunta. Servicios para la prevención y tratamiento de los trastornos genéticos y los defectos congénitos en los países en desarrollo. La Haya; 1999. (WHO/HGN/GL/ WAOPBD/99.1).

2. Organización Panamericana de la Salud. Informe de un Grupo de Consulta. Prevención y control de las enfermedades genéticas y los defectos congénitos. Washington, D.C.: OPS; 1984. (Publicación Científica 460).

3. Word Health Organization. Report of a WHO Scientific Group. Control of hereditary dis- eases. Geneva: WHO; 1996. (Technical Report Series 865).

4. Council of Regional Networks for Genetic Services. Guidelines for clinical genetic services for the public's health, 1st ed. Atlanta: CORN; 1997.

5. Beiguelman B, Penchaszadeh VB, eds. Servicos de genética médica na América Latina: Estado atual e perspectivas. Brazilian J Genet. 1997; 20(suppl 1):1-175.

6. Harris R, Reid M. Medical genetic services in 31 countries: an overview. Eur J Hum Genet. 1997; 5(suppl 2):3-21.

7. Word Health Organization. Report of the Advisory Committee on Health Research. Ge- nomics and World Health. Geneva: WHO 2002.

8. Penchaszadeh VB. Delivery of genetic services in developing countries. En: Khoury M, Burke W, Thompson E, eds. Genetics and public health in the 21st Century. Oxford University Press; 2000.

9. Word Health Organization. Report of a WHO Consultation. Community genetic services in Latin America and regional networks on medical genetics. Porto Alegre: WHO; 2003.

10. Organización Panamericana de la Salud. Situación de la salud en las Américas. Indicadores básicos: Argentina. Washington, D.C.: OPS; 2004. 
11. Barreiro C. Situación de los servicios de genética médica en Argentina. Brazilian J Genet. 1997;20(supl 1):5-10.

12. Emery AEH, Rimon DL. Principles and practice of medical genetics. Churchill Livingstone: London; 2002.

13. Khrouf N, Spang R, Podgorna T, Miled SB, Moussaoui M, Chibani M. Malformations in 10,000 consecutive births in Tunis. Acta Paediatr Scand. 1986;75:534-9.
14. Castilla EE, Orioli IM. ECLAMC: The LatinAmerican Collaborative Study of Congenital Malformations. Community Genetics. 2004;7: 76-94.

15. Castilla EE, López Camelo JS, Paz JE. Atlas Geográfico de las Malformaciones Congénitas en Sudamérica. Río de Janeiro: Editora Fiocruz; 1995.

16. Argentina. Programa Nacional de Estadísticas de Salud. Estadísticas vitales. Buenos Aires:
Ministerio de Salud y Ambiente de la Nación; 2003.

Manuscrito recibido el 28 de junio de 2005. Aceptado para publicación, tras revisión, el 17 de noviembre de 2005.

ABSTRACT Objective. To describe the medical genetics services in Argentina, in order to help organize a network to coordinate those services, decrease inequity in access to them, and promote the comprehensive development of medical genetics in the country.

A census of medical genetics Methods. In April-August 2004 a survey was conducted of centers providing medservices in Argentina

ical genetics services in Argentina, looking at their human resources, diagnostic procedures, the services that patients use, and funding. The survey covered service centers that conduct genetic assessment, cytogenetic diagnostic testing, molecular biology testing, and biochemical testing to detect birth defects or genetic disorders. Centers that were devoted exclusively to research were not surveyed.

Results. A total of 134 centers fulfilled the selection criteria for the survey; 84 of them were private services, and the other 50 were publicly managed services, at universities and hospitals. The 50 public facilities had a total of 67 physicians who had studied clinical genetics, plus 133 non-medical laboratory professionals. A majority of the 50 centers performed clinical and cytogenetic diagnoses, with a smaller number performing molecular, biochemical, and prenatal diagnoses. More than $70 \%$ of the centers and human resources were located in an area that includes the city of Buenos Aires, the province of Buenos Aires, and the nearby north-central provinces of Córdoba and Santa Fe. The 50 public centers were found mainly in large cities; 10 of the country's 23 provinces had no public services.

Conclusions. There is a great geographic disparity in the availability of resources as well as wide variability in the diagnostic procedures that are provided in the different service centers. The unequal distribution of the facilities is an organizational problem that will require the application of policies to guarantee minimum services for people in all regions of the country, as well as access to more complex diagnostic procedures.

Key words Genetics; health care facilities, manpower, and services; Argentina. 